\title{
Risk Factors of Lymph Node Metastasis in Patients with Early Pure and Mixed Signet Ring Cell Gastric Carcinomas
}

\author{
Qingqing Hu1\#, Raymond Dekusaah²\#, Shouli Cao1, Taohong Pang11, Yi Wang1, Bin Zhang1, Ying Lv1, Xiaoqi \\ Zhang $^{1}$, Tingsheng Ling ${ }^{1}$, Yuzheng Zhuge ${ }^{1}$, Lei Wang, ${ }^{1}$ Xiaoping Zou ${ }^{1}$, Weijie Zhang ${ }^{\circledR}$, Qin Huang ${ }^{1,4^{\bowtie}}$, \\ Guifang $\mathrm{Xu}^{1,2}$
}

1. Department of Gastroenterology, Affiliated Drum Tower Hospital of Nanjing University Medical School, Nanjing, China

2. Department of Geriatric, Drum Tower Clinical College of Nanjing Medical University, Nanjing, China

3. Department of General Surgery, Affiliated Drum Tower Hospital of Nanjing University Medical School, Nanjing, China

4. VA Boston Healthcare System and Harvard Medical School, West Roxbury, MA 02132, USA.

\# These authors contributed equally to the work.

$\triangle$ Corresponding authors: Dr. Guifang Xu, The Affiliated Drum Tower Hospital of Nanjing University Medical School, 321 Zhongshan Road, Nanjing, Jiangsu 210008, China. Email: 13852293376@163.com. Telephone: +86-25-83105206 Fax: +86-25-83105206. Dr. Qin Huang, VA Boston Healthcare System and Harvard Medical School, West Roxbury, MA 02132, USA. Email: qinhuang0122@gmail.com. Dr. Weijie Zhang, The Affiliated Drum Tower Hospital of Nanjing University Medical School, 321 Zhongshan Road, Nanjing, Jiangsu 210008, China. Email: 13770773971@163.com. Telephone: +86-25-83105206 Fax: +86-25-83105206.

(C) Ivyspring International Publisher. This is an open access article distributed under the terms of the Creative Commons Attribution (CC BY-NC) license (https://creativecommons.org/licenses/by-nc/4.0/). See http://ivyspring.com/terms for full terms and conditions.

Received: 2018.08.15; Accepted: 2019.01.02; Published: 2019.01.29

\begin{abstract}
Background: Early gastric carcinoma (EGC) with pure signet ring cell carcinoma (PSRCC) has been reported to have favourable prognosis and low risk of lymph node metastasis (LNM). However, risk factors of LNM and clinicopathological features for early gastric mixed signet ring cell carcinoma (mSRCC) remain poorly investigated. The aim of this study was to identify risk factors of LNM and compare clinicopathological characteristics and prognosis of early gastric PSRCC with $\mathrm{mSRCC}$.

Methods: This retrospective study was conducted at our center between 2005 and 2015 in 796 patients underwent radical gastrectomies combined with lymph node dissections, A total of 160 patients with early gastric SRCC underwent gastrectomies with lymph node dissections were reviewed, in which 79 cases were PSRCC and 81 cases were mSRCC. Risk factors of LNM and clinicopathologic features of these two groups were statistically compared, including age, gender, tumor location, gross pattern, size, invasion depth, lymphovascular invasion (LVI), helicobacter pylori $(H p)$ infection, atrophic gastritis, ulcer finding and LNM. Patients were follow-up for post-resection survival. The 5-year survival and disease-specific survival rate were estimated with the Kaplain-Meier method with a log-rank test and compared between the two groups.

Results: Tumor size $(P<0.05)$, invision depth $(P<0.05)$ and LVI $(P<0.0001)$ were risk factors of LNM, LVI $(P<$ $0.0001)$ was independent risk factor of LNM in 160 patients. Univariate analysis reviewed LVI $(P<0.0001)$ as the risk factor in the PSRCC group, and the risk factors of LNM in the mSRCC included LVI $(P<0.0001)$ and tumor size $(P<0.05)$. Multivariable analysis revealed two independent risk factors in the mSRCC group: 1$)$ tumor size $(P<0.05)$, and 2$)$ LVI $(P<0.0001)$. The significant characteristics in two groups included the male gender $(P<0.0001)$, gross pattern $(P<0.05)$, LVI $(P<0.01)$, and $H$ p infection $(P<0.01)$. The difference of LNM rate between expanded indication and out of indication in 160 patients was significant $(P=0.03)$. The overall 5 -year survival rate for early gastric SRCC was $96.3 \%$. There was no significant difference in the overall survival and disease-specific survival between the two groups.

Conclusions: Although with similar post-resection survival, the independent risk factors of LNM in the early mSRCC group, compared to those in the early PSRCC group, included large tumor size and LVI. Early gastric mSRCC had more aggressive clinicopathological features than PSRCC.
\end{abstract}

Key words: Early gastric carcinoma (EGC), Pure signet ring cell cancer (pSRCC), Mixed signet ring cell cancer (mSRCC), Lymph node metastasis(LNM) 


\section{Introduction}

Gastric cancer remains one of leading cancer in incidence and mortality in the world, especially for SRCC that is usually fatal because of wide-spread LNM and distant metastasis [1]. However, patients with EGC resection would have a much better prognosis with the 5-year survival rate of over $90 \%$, as reported in China, Japan, and Western countries [2]. The standard clinical practice guidelines for treatment of EGC include radical gastrectomy with nodal dissection [3]. Recent studies reveal a very low risk of LNM in EGC, including early gastric SRCC [4], suggesting a significant role of endoscopic resection in EGC therapy with the endoscopic techniques such as endoscopic mucosa resection (EMR) and endoscopic submucosal dissection (ESD) compared with surgical resection, endoscopic therapy has the same long-term therapeutic effect, better quality of life [5], and excellent prognosis [6-8].

Clinical decision on endoscopic, vs, surgical resection of EGC relies upon an accurate assessment of risk of LNM. Overall, tumor size, histological type, LVI, and depth of invasion are independent risk factors for LNM in EGC [9], while risk factors of LNM for early pSRCC and mSRCC stay elusive. Accordig to the World Health Organization (WHO) diagnostic criteria [10], SRCC was classified as part of gastric adenocarcinoma with dismal prognosis. Recently, Zheng et al. [11] reported that mixed gastric cancer has more aggressive features than the pure Lauren intestinal-type or diffuse-type gastric cancer, because of deeper infiltration, greater dimension, and more frequent LNM. However, the published studies on clinicopathological features and prognosis of early mSRCC are scarce.

The purpose of the present study was to investigate risk factors of LNM, clinicopathological characteristics, and prognosis of early gastric pSRCC and mSRCC to guide a better precision therapeutic strategy.

\section{Methods}

\section{Patient selection and groups}

A total of consecutive 796 patients were identified with radical gastrectomy and lymph node dissection for EGC at the Division of Digestive Surgery, Department of Surgery, the Nanjing Drum Tower Hospital over the period between 2005 and 2015. Each pathology report was reviewed for pathologic diagnosis on the basis of the WHO diagnostic criteria [10]. In the cohort of 796 EGCs, 79 $(9.9 \%)$ cases were diagnosed as pSRCC and 81 (10.2\%) as mSRCC. This study was approved by the Ethics
Committee of the Affiliated Drum Tower Hospital Affiliated to Nanjing University.

\section{Clinicopathological analysis}

Electronic patient medical records of each selected patient were reviewed for demographic information, medical history, surgical note, pathological report, postoperative hospital course, and therapeutic outcomes. Resected specimens were routinely processed with a standard pathology gastric cancer resection protocol. After gross examination, the specimen was transversely sectioned at the interval of $4 \mathrm{~mm}$ in width, embedded in paraffin, and routinely stained with hematoxylin and eosin. Two experienced pathologists independently retrieved and investigated each case blindly without the knowledge of clinical and endoscopic findings, several specialists discussed and reached a consensus in difficult cases.

The WHO diagnostic critiera for EGC was followed. As such, pSRCC was defined as a predominant component $(>50 \%)$ isolated carcinoma cells containing intracytoplasmic mucin, and mSRCC was defined as adenocarcinoma with a minor component $(10-50 \%)$ of isolated carcinoma cells containing intracytoplasmic mucin [10]. Tumor location, maximal size, gross pattern (on the basis of the Paris classification) [12], invasion depth, which was divided as M (intramucosal) and SM (submucosal invasion), and LVI were also recorded. The total numbers of lymph nodes retrieved and involved by carcinoma in each case were abstracted from the pathology report. Poor differentiation as indiscernible tubules and glands in less than $50 \%$ of tumor on a section, and moderate differentiation with discernible glands/tubules between $50 \%$ and $95 \%$, based on the Vienna criteria [13]. Ulceration was defined as rupture of the muscularis mucosae and fibrosis in the SM layer by histological findings. Hp infection status was determined by a rapid urease test or more of the tests of biopsy specimens and any positive result defined $\mathrm{Hp}$ infection. The definition of atrophic gastritis is based on the criteria of the updated Sydney System for the classification of Gastritis [14]. The definitions of expanded indication and out of indication were based on the Japanese classification of gastric carcinoma [Japanese Gastric Cancer Association, 2011].

\section{Post-resection survival}

Post-resection outcomes were investigated through routine scheduled in-office visits, which were documented in the patient electronic medical record. Telephone interview were performed routinely at 6 , 12 , and 18 months, and then annually after the surgery to assess the general situation of each patient 
by the investigators. Patient post-resection survival was calculated from the day of surgery to the day of the last follow-up interview or the day of death of any causes.

\section{Statistical Analysis}

Statistical analyses were carried out with SPSS 22.0 (IBM, Armonk, New York, USA). Quantitative data were expressed as mean \pm standard deviation (SD). Absolute and relative frequencies for categorical variables were analyzed with rate. The two-tailed Student's $t$ and the Mann Whitney $U$ tests were used for analysis of normally distributed and non-normally continuous variables, respectively. Univariate analysis of risk factors of LNM and the relationship with clinicopathological features was assessed by the Chi-square or Fisher exact test. Multivariate analysis by a logistic regression model was employed to investigate independent risk factors of LNM. Patient's overall survival was estimated by the Kaplan - Meier method with a log-rank test. A $P$ value of $<0.05$ was considered statistically significant.

\section{Results}

\section{Demographic and clinicopathological characteristics}

Baseline and clinicopathological characteristics of 160 patients were summarized in Table 1 . The mean age was 53.1years, and the male-to-female ratio was 1.4:1. Gastric body and gastric antrum were $31.9 \%$ and $40.0 \%$, respectively. Depressed type (0-III gross pattern) was found in 65 patients $(40.0 \%)$ and 40 lesions $(25.0 \%)$ were IIc gross pattern. The proportion of patients with tumor size over $2 \mathrm{~cm}$ was $45 \%$. Mucosal and submucosal lesions were $58.1 \%$ and $41.9 \%$, respectively. Of these 160 patients, $16(10.0 \%)$ patients had Lymphovascular invasion, $72(45.0 \%)$ patients had $\mathrm{Hp}$ infection, $87(54.4 \%)$ patients occured atrophic gastritis and 92 (57.5\%) patients had Ulcer findings.

\section{Risk factors of lymph node metastasis}

Univarite analysis showed that tumor $\operatorname{size}(P<$ $0.05)$, invasion depth $(P<0.05)$ and LVI $(P<0.0001)$ were risk factors of LNM with the total 160 SRCCs in Table 2, multivariate analysis revealed LVI with the odds ratio of 25.6 was the significant independent risk factor of LNM (95\% confidence interval: 6.7 - 98.7) $(P$ $<0.0001$ ). By univariate analysis (Table 3 ), significant risk factor of LNM in pSRCC was LVI $(P<0.0001)$, in which 2 cases of LNM all appeared LVI (100\%). Multivariate analysis failed to find its independent risk factors.

In the mSRCC group, significant risk factors of LNM included LVI $(P<0.0001)$ and tumor size of $>$
$1.0 \mathrm{~cm}(P<0.05)$; no LNM was discovered in tumors with the size of $<1.0 \mathrm{~cm}$. None of other risk factors of LNM were statistically significant, as shown in Table 4. The multivariate analysis revealed two significant independent risk factors of LNM in MSRCC: 1 ) tumor size with the odds ratio of 2.1 (95\% confidence interval: 1.0 - 4.1) $(P<0.05)$, and 2) LVI with the odds ratio of 22.2 (95\% confidence interval: 4.8 - 103.1) $(P<$ 0.0001) (Table 3).

Table 1. Demographic and clinical characteristics in 160 patients with early pure and mixed signet-ring cell gastric carcinoma.

\begin{tabular}{|c|c|}
\hline Age (year) & \\
\hline median (range) & $52(20-86)$ \\
\hline mean & 53.1 \\
\hline Gender & \\
\hline Male & $93(58.1 \%)$ \\
\hline Female & $67(41.9 \%)$ \\
\hline Location & \\
\hline cardia & $11(6.9 \%)$ \\
\hline body & $51(31.9 \%)$ \\
\hline angularis & $19(11.9 \%)$ \\
\hline antrum & $64(40.0 \%)$ \\
\hline pylorus & $15(9.4 \%)$ \\
\hline Gross pattern & \\
\hline $0-\mathrm{I}$ & $3(1.9 \%)$ \\
\hline $0-2 a$ & $19(11.9 \%)$ \\
\hline $0-2 b$ & $33(20.6 \%)$ \\
\hline $0-2 c$ & $40(25.0 \%)$ \\
\hline $0-3$ & $65(40.6 \%)$ \\
\hline Tumor size(cm) & \\
\hline$\leq 2.0$ & $88(55.0 \%)$ \\
\hline$>2.0$ & $72(45.0 \%)$ \\
\hline Invasion depth & \\
\hline M & 93(58.1\%) \\
\hline SM & $67(41.9 \%)$ \\
\hline Lymphovascular & \\
\hline Absence & $144(90.0 \%)$ \\
\hline Presence & $16(10.0 \%)$ \\
\hline Hp infection & \\
\hline Absence & $88(55.0 \%)$ \\
\hline Presence & $72(45.0 \%)$ \\
\hline Atrophic gastriti & \\
\hline Absence & $73(45.6 \%)$ \\
\hline Presence & $87(54.4 \%)$ \\
\hline Histological typ & \\
\hline Pure & $79(49.4 \%)$ \\
\hline Mixed & $81(50.6 \%)$ \\
\hline Ulcer finding & \\
\hline Absence & $68(42.5 \%)$ \\
\hline Presence & $92(57.5 \%)$ \\
\hline
\end{tabular}

M: intramucosal; SM: submucosal invasion; $H p$ : helicobacter pylori.

\section{Comparison of clinicopathological features between PSRCC and $\mathrm{MSRCC}$}

As shown in Table 5, differences in clinicopathological characteristics between pSRCC and mSRCC groups were significant for the followings: 1) gender was much higher in mSRCC $(74.1 \%)$ than in pSRCC $(41.8 \%)(P<0.0001) ; 2)$ tumor gross pattern that demonstrated a much higher percentage cases with ulcer (pattern 0-III) in mSRCC $(51.9 \%)$ than in pSRCC $(29.1 \%)$, while the frequencies in flat (pattern 0-IIb) and slightly depressed (pattern 
0-IIc) gross patterns were lower in mSRCC than in pSRCC $(P<0.05)$; 3$)$ LVI that showed a significantly higher proportion of cases in mSRCC $(17.3 \%)$ than in pSRCC $(2.5 \%)(P<0.01)$; 4$) \mathrm{Hp}$ infection that was much more frequent in mSRCC $(56.8 \%)$ than in pSRCC $(32.9 \%)(P<0.01)$. However, there was no significant difference in age, location, size, invasion depth, atrophic gastritis, and ulcerative findings between the mSRCC and pSRCC groups. More frequent LNM was observed in the mSRCC (27.2\%) than in the pSRCC $(13.9 \%)$ groups, although the difference was not statistically significant.

Table 2. Univariate and Multivariate analysis of risk factors for lymph node metastasis in 160 patients with early pure and mixed signet-ring cell gastric carcinoma.

\begin{tabular}{|c|c|c|c|c|c|}
\hline \multicolumn{6}{|c|}{ Univariate analysis of risk factors for LNM in 160 patients with early gastric SRCC } \\
\hline \multirow[t]{2}{*}{$\begin{array}{l}\text { Clinicopathologic } \\
\text { Feature }\end{array}$} & \multirow[t]{2}{*}{$\begin{array}{l}\text { Total } \\
\text { Number }\end{array}$} & \multicolumn{2}{|c|}{$\begin{array}{l}\text { Lymph Node } \\
\text { Metastasis }\end{array}$} & \multirow[t]{2}{*}{ Percent } & \multirow[t]{2}{*}{ P Value } \\
\hline & & Absence & Presence & & \\
\hline \multicolumn{6}{|l|}{ Age (year) } \\
\hline$\leq 65$ & 133 & 103 & 30 & 22.6 & \multirow[t]{2}{*}{ NS } \\
\hline$>65$ & 27 & 24 & 3 & 11.1 & \\
\hline \multicolumn{6}{|l|}{ Gender } \\
\hline Male & 93 & 74 & 19 & 20.4 & \multirow[t]{2}{*}{ NS } \\
\hline Female & 67 & 53 & 14 & 20.9 & \\
\hline \multicolumn{6}{|l|}{ Location } \\
\hline cardia & 11 & 9 & 2 & 18.2 & \multirow[t]{5}{*}{ NS } \\
\hline body & 51 & 42 & 9 & 17.6 & \\
\hline angularis & 19 & 17 & 2 & 10.5 & \\
\hline antrum & 64 & 48 & 16 & 25.0 & \\
\hline pylorus & 15 & 11 & 4 & 26.7 & \\
\hline \multicolumn{6}{|l|}{ Gross pattern } \\
\hline $0-\mathrm{I}$ & 3 & 3 & 0 & 0.0 & \multirow[t]{5}{*}{ NS } \\
\hline $0-2 \mathrm{a}$ & 19 & 15 & 4 & 21.1 & \\
\hline $0-2 b$ & 33 & 27 & 6 & 18.2 & \\
\hline $0-2 \mathrm{c}$ & 40 & 31 & 9 & 22.5 & \\
\hline $0-3$ & 65 & 51 & 14 & 21.5 & \\
\hline \multicolumn{6}{|l|}{ Tumor size(cm) } \\
\hline$\leq 2.0$ & 88 & 76 & 12 & 13.6 & \multirow[t]{2}{*}{$<0.05$} \\
\hline$>2.0$ & 72 & 51 & 21 & 29.2 & \\
\hline \multicolumn{6}{|l|}{ Invasion depth } \\
\hline M & 93 & 79 & 14 & 15.1 & \multirow[t]{2}{*}{$<0.05$} \\
\hline SM & 67 & 48 & 19 & 28.4 & \\
\hline \multicolumn{6}{|l|}{$\begin{array}{l}\text { Lymphovascular } \\
\text { invasion }\end{array}$} \\
\hline Absence & 144 & 124 & 20 & 13.9 & \multirow[t]{2}{*}{$<0.0001$} \\
\hline Presence & 16 & 3 & 13 & 81.3 & \\
\hline \multicolumn{6}{|l|}{$H p$ infection } \\
\hline Absence & 88 & 69 & 19 & 27.5 & \multirow[t]{2}{*}{ NS } \\
\hline Presence & 72 & 58 & 14 & 19.4 & \\
\hline \multicolumn{6}{|l|}{ Atrophic gastritis } \\
\hline Absence & 73 & 61 & 12 & 16.4 & NS \\
\hline Presence & 87 & 66 & 21 & 24.1 & \\
\hline Histological type & & & & & \\
\hline Pure & 79 & 68 & 11 & 13.9 & NS \\
\hline Mixed & 81 & 59 & 22 & 27.2 & \\
\hline Ulcer finding & & & & & \\
\hline Absence & 68 & 50 & 18 & 26.5 & NS \\
\hline Presence & 92 & 77 & 15 & 16.3 & \\
\hline $\begin{array}{l}\text { Multivariate analys } \\
\text { SRCC }\end{array}$ & risk facto & for LNM in 1 & 60 patients & vith early & \\
\hline & $P$ value & Odds ratio & $95 \%$ Con & dence int & vals \\
\hline $\begin{array}{l}\text { Lymphovascular } \\
\text { invasion }\end{array}$ & $<0.0001$ & 25.6 & $6.7-98.7$ & & \\
\hline Tumor size & NS & 2.4 & $1.0-6.0$ & & \\
\hline Invasion depth & NS & 1.0 & $0.3-2.5$ & & \\
\hline
\end{tabular}

NS: not significant; SRCC: signet-ring cell gastric carcinoma; $H p$ : helicobacter pylori; LNM: lymph node metastasis; M: intramucosal; SM: submucosal invasion.
Table 3. Univariate analysis of risk factors for lymph node metastasis in 79 patients with early pure signet-ring cell gastric carcinoma.

\begin{tabular}{|c|c|c|c|c|c|}
\hline \multirow[t]{2}{*}{$\begin{array}{l}\text { Clinicopathologic } \\
\text { Feature }\end{array}$} & \multirow[t]{2}{*}{$\begin{array}{l}\text { Total } \\
\text { Number }\end{array}$} & \multicolumn{2}{|c|}{$\begin{array}{l}\text { Lymph Node } \\
\text { Metastasis }\end{array}$} & \multirow[t]{2}{*}{ Percent } & \multirow[t]{2}{*}{$P$ Value } \\
\hline & & Absence & Presence & & \\
\hline \multicolumn{6}{|l|}{ Age (year) } \\
\hline$\leq 65$ & 69 & 58 & 11 & 15.9 & \multirow[t]{2}{*}{ NS } \\
\hline$>65$ & 10 & 10 & 0 & 0 & \\
\hline \multicolumn{6}{|l|}{ Gender } \\
\hline Male & 33 & 29 & 4 & 12.1 & \multirow[t]{2}{*}{ NS } \\
\hline Female & 46 & 39 & 7 & 15.2 & \\
\hline \multicolumn{6}{|l|}{ Location } \\
\hline cardia & 4 & 3 & 1 & 25.0 & \multirow[t]{5}{*}{ NS } \\
\hline body & 23 & 20 & 3 & 13.0 & \\
\hline angularis & 10 & 9 & 1 & 10.0 & \\
\hline antrum & 32 & 28 & 4 & 12.5 & \\
\hline pylorus & 10 & 8 & 2 & 20.0 & \\
\hline \multicolumn{6}{|l|}{ Gross pattern } \\
\hline $0-\mathrm{I}$ & 2 & 2 & 0 & 0.0 & \multirow[t]{5}{*}{ NS } \\
\hline $0-2 a$ & 9 & 8 & 1 & 11.1 & \\
\hline $0-2 b$ & 21 & 18 & 3 & 14.3 & \\
\hline $0-2 c$ & 24 & 20 & 4 & 16.7 & \\
\hline $0-3$ & 23 & 20 & 3 & 13.0 & \\
\hline \multicolumn{6}{|l|}{ Tumor size(cm) } \\
\hline$\leq 2.0$ & 49 & 43 & 6 & 12.2 & \multirow[t]{2}{*}{ NS } \\
\hline$>2.0$ & 30 & 25 & 5 & 16.7 & \\
\hline \multicolumn{6}{|l|}{ Invasion depth } \\
\hline $\mathrm{M}$ & 51 & 45 & 6 & 11.8 & \multirow[t]{2}{*}{ NS } \\
\hline $\mathrm{SM}$ & 28 & 23 & 5 & 17.9 & \\
\hline \multicolumn{6}{|l|}{$\begin{array}{l}\text { Lymphovascular } \\
\text { invasion }\end{array}$} \\
\hline Absence & 77 & 68 & 9 & 11.7 & \multirow[t]{2}{*}{$<0.0001$} \\
\hline Presence & 2 & 0 & 2 & 100.0 & \\
\hline \multicolumn{6}{|l|}{$H p$ infection } \\
\hline Absence & 53 & 45 & 8 & 15.1 & \multirow[t]{2}{*}{ NS } \\
\hline Presence & 26 & 23 & 3 & 11.5 & \\
\hline \multicolumn{6}{|l|}{ Atrophic gastritis } \\
\hline Absence & 39 & 33 & 6 & 15.4 & \multirow[t]{2}{*}{ NS } \\
\hline Presence & 40 & 35 & 5 & 12.5 & \\
\hline \multicolumn{6}{|l|}{ Ulcer finding } \\
\hline Absence & 35 & 30 & 5 & 14.3 & \multirow[t]{2}{*}{ NS } \\
\hline Presence & 44 & 38 & 6 & 13.6 & \\
\hline
\end{tabular}

In the pSRCC group, LNM rate in patients with expanded indication was $8.7 \%(2 / 23)$, and out of indication was $16.1 \%(9 / 56)$. In the mSRCC group, LNM rate in patients with expanded indication was $0(0 / 8)$, and out of indication was $30.1 \%(22 / 73)$. There was no statistical difference between the above two groups ( $P=0.32$ and $P=0.06$, respectively). In all 160 patients, LNM rate between the expanded indication $(2 / 31,6.5 \%)$ and out of indication $(31 / 129,24 \%)$ had statistically significant differences $(P=0.03)$ (Table 6). The clinicopathological characteristics in 2 cases with LNM for expanded indication were showed in Table 7.

\section{Post-resection survival}

At the last follow-up interview of 160 patients in the cohort, $46(28.8 \%)$ patients were lost and 114 $(71.2 \%)$ patients completed the 5 year survival survey. 
The overall average survival time for the cohort was 62 months (range: 6-153). The 5-year survival rate was $96.3 \%$. In the mSRCC group, 54 (66.7\%, 54/81) completed the follow up and 3 patients died, two of whom died of the disease and one for other cause. In the pSRCC group, $60(75.9 \%, 60 / 79)$ had the survival information with 3 deaths for unrelated diseases. There was no statistically significant difference in the average survival months between mSRCC $(58.5+25.2$ months) and pSRCC $(66.7+26.9$ months $)$ groups $(P>$ 0.05 ) (Figure 1). No significant difference in disease-specific survival was observed between the two groups $(P>0.05)$ (Figure 2$)$.

Table 4. Univariate and Multivariate analysis of risk factors for lymph node metastasis in 81 patients with early mixed signet-ring cell gastric carcinoma

\begin{tabular}{|c|c|c|c|c|c|}
\hline \multicolumn{6}{|c|}{ Univariate analysis of risk factors for LNM in 81patients with early gastric mSRCC } \\
\hline \multirow{3}{*}{$\begin{array}{l}\text { Clinicopathologic } \\
\text { Feature }\end{array}$} & \multirow{3}{*}{$\begin{array}{l}\text { Total } \\
\text { Number }\end{array}$} & \multirow{2}{*}{\multicolumn{2}{|c|}{$\begin{array}{l}\text { Lymph Node } \\
\text { Metastasis }\end{array}$}} & \multirow{3}{*}{$\begin{array}{l}\text { Percent } \\
(\%)\end{array}$} & \multirow{3}{*}{$\begin{array}{l}P \\
\text { Value }\end{array}$} \\
\hline & & & & & \\
\hline & & Absence & Presence & & \\
\hline \multicolumn{6}{|l|}{ Age (year) } \\
\hline$\leq 65$ & 64 & 45 & 19 & 29.7 & \multirow[t]{2}{*}{ NS } \\
\hline$>65$ & 17 & 14 & 3 & 17.6 & \\
\hline \multicolumn{6}{|l|}{ Gender } \\
\hline Male & 60 & 45 & 15 & 25.0 & \multirow[t]{2}{*}{ NS } \\
\hline Female & 21 & 14 & 7 & 33.3 & \\
\hline \multicolumn{6}{|l|}{ Location } \\
\hline cardia & 7 & 6 & 1 & 14.3 & \multirow[t]{5}{*}{ NS } \\
\hline body & 28 & 22 & 6 & 21.4 & \\
\hline angularis & 9 & 8 & 1 & 11.1 & \\
\hline antrum & 32 & 20 & 12 & 37.5 & \\
\hline pylorus & 5 & 3 & 2 & 40.0 & \\
\hline \multicolumn{6}{|l|}{ Gross pattern } \\
\hline $0-\mathrm{I}$ & 1 & 1 & 0 & 0.0 & \multirow[t]{5}{*}{ NS } \\
\hline $0-2 a$ & 10 & 7 & 3 & 30.0 & \\
\hline $0-2 b$ & 12 & 9 & 3 & 25.0 & \\
\hline $0-2 \mathrm{c}$ & 16 & 11 & 5 & 31.3 & \\
\hline $0-3$ & 42 & 31 & 11 & 26.2 & \\
\hline \multicolumn{6}{|l|}{ Tumor size(cm) } \\
\hline$\leq 2.0$ & 39 & 33 & 6 & 15.4 & \multirow[t]{2}{*}{$<0.05$} \\
\hline$>2.0$ & 42 & 26 & 16 & 38.1 & \\
\hline \multicolumn{6}{|l|}{ Invasion depth } \\
\hline $\mathrm{M}$ & 42 & 34 & 8 & 19.0 & \multirow[t]{2}{*}{ NS } \\
\hline SM & 39 & 25 & 14 & 35.9 & \\
\hline \multicolumn{6}{|l|}{$\begin{array}{l}\text { Lymphovascular } \\
\text { invasion }\end{array}$} \\
\hline Absence & 67 & 56 & 11 & 16.4 & \multirow[t]{2}{*}{$<0.0001$} \\
\hline Presence & 14 & 3 & 11 & 78.6 & \\
\hline \multicolumn{6}{|l|}{ Hp infection } \\
\hline Absence & 35 & 24 & 11 & 31.4 & \multirow[t]{2}{*}{ NS } \\
\hline Presence & 46 & 35 & 11 & 23.9 & \\
\hline \multicolumn{6}{|l|}{ Atrophic gastritis } \\
\hline Absence & 34 & 28 & 6 & 17.6 & NS \\
\hline Presence & 47 & 31 & 16 & 34.0 & \\
\hline Ulcer finding & & & & & \\
\hline Absence & 33 & 20 & 13 & 39.4 & NS \\
\hline Presence & 48 & 39 & 9 & 18.8 & \\
\hline $\begin{array}{l}\text { Mixed pathologica } \\
\text { type }\end{array}$ & & & & & \\
\hline $\mathrm{p} / \mathrm{D}$ & 62 & 45 & 17 & 27.4 & NS \\
\hline $\mathrm{m} / \mathrm{D}$ & 19 & 14 & 5 & 26.3 & \\
\hline $\begin{array}{l}\text { Multivariate analys } \\
\text { mSRCC }\end{array}$ & risk factor & r LNM in 81 & patients wit & early ga & \\
\hline & $P$ value & Odds ratio & $95 \%$ Confi & dence inte & rals \\
\hline Tumor size & $<0.05$ & 2.1 & $1.0-4.1$ & & \\
\hline $\begin{array}{l}\text { Lymphovascular } \\
\text { invasion }\end{array}$ & $<0.0001$ & 22.2 & $4.8-103.1$ & & \\
\hline
\end{tabular}

NS: not significant; $p$ /D:poorly differentiated adenocarcinoma; m/D:middle differentiated adenocarcinoma; mSRCC: mixed signet-ring cell carcinoma; $\mathrm{Hp}$ helicobacter pylori; LNM: lymph node metastasis; M: intramucosal; SM: submucosal invasion.

Table 5. Comparison of clinicopathological characteristics between early pure and mixed signet-ring cell gastric carcinoma

\begin{tabular}{|c|c|c|c|}
\hline Clinicopathologic Feature & Mixed SRCC & Pure SRCC & $P$ value \\
\hline \multicolumn{4}{|l|}{ Age (year) } \\
\hline mean $\pm S D$ & $54.8 \pm 12.0$ & $51.3 \pm 13.0$ & NS \\
\hline median(range) & $54(20-69)$ & $51.5(27-69)$ & \\
\hline \multicolumn{4}{|l|}{ Gender } \\
\hline Male & $60(74.1)$ & $33(41.8)$ & $<0.0001$ \\
\hline Female & $21(25.9)$ & $46(58.2)$ & \\
\hline \multicolumn{4}{|l|}{ Location } \\
\hline cardia & $7(8.6)$ & $4(5.1)$ & NS \\
\hline body & $28(34.6)$ & $23(29.1)$ & \\
\hline angularis & $9(11.1)$ & $10(12.7)$ & \\
\hline antrum & $32(39.5)$ & $32(40.5)$ & \\
\hline pylorus & $5(6.2)$ & $10(12.7)$ & \\
\hline \multicolumn{4}{|l|}{ Gross pattern } \\
\hline $0-\mathrm{I}$ & $1(1.2)$ & $2(2.5)$ & $<0.05$ \\
\hline $\mathrm{O}-2 \mathrm{a}$ & $10(12.3)$ & $9(11.4)$ & \\
\hline $\mathrm{O}-2 \mathrm{~b}$ & $12(14.8)$ & $21(26.6)$ & \\
\hline $\mathrm{O}-2 \mathrm{c}$ & $16(19.8)$ & $24(30.4)$ & \\
\hline O-3 & $42(51.9)$ & $23(29.1)$ & \\
\hline \multicolumn{4}{|l|}{ Tumor size(cm) } \\
\hline mean $\pm \mathrm{SD}$ & $2.5 \pm 1.3$ & $2.3 \pm 1.6$ & NS \\
\hline median(range) & $2.2(0.4-3.5)$ & $2(0.2-6.5)$ & \\
\hline \multicolumn{4}{|l|}{ Invasion depth } \\
\hline $\mathrm{M}$ & $42(51.8)$ & $51(64.5)$ & NS \\
\hline $\mathrm{SM}$ & $39(48.2)$ & $28(35.5)$ & \\
\hline \multicolumn{4}{|l|}{ Lymphovascular invasion } \\
\hline Absence & $67(82.7)$ & $77(97.5)$ & $<0.01$ \\
\hline Presence & 14(17.3) & $2(2.5)$ & \\
\hline \multicolumn{4}{|l|}{$H p$ infection } \\
\hline Absence & $35(43.2)$ & $53(67.1)$ & $<0.01$ \\
\hline Presence & $46(56.8)$ & $26(32.9)$ & \\
\hline \multicolumn{4}{|l|}{ Atrophic gastritis } \\
\hline Absence & $34(42.0)$ & $39(49.4)$ & NS \\
\hline Presence & $47(58.0)$ & $40(50.6)$ & \\
\hline \multicolumn{4}{|l|}{ Lymph node metastasis } \\
\hline Absence & $59(72.8)$ & $68(86.1)$ & NS \\
\hline Presence & $22(27.2)$ & 11(13.9) & \\
\hline \multicolumn{4}{|l|}{ Ulcer finding } \\
\hline Absence & $33(40.7)$ & $35(44.3)$ & NS \\
\hline Presence & $48(59.3)$ & $44(55.7)$ & \\
\hline
\end{tabular}

Table 6. Comparison of lymph node metastasis rate between expanded indication and out of indication.

\begin{tabular}{lllllllll}
\hline & \multicolumn{3}{c}{ Expanded indication } & \multicolumn{3}{c}{ Out of indication } & P Value \\
\cline { 2 - 7 } & Total & LNM & \%LNM & Total & LNM & \%LNM & \\
\hline Pure SRCC & 23 & 2 & 8.7 & 56 & 9 & 16.1 & 0.32 \\
Mixed SRCC & 8 & 0 & 0 & 73 & 22 & 30.1 & 0.06 \\
Pure and mixed SRCC & 31 & 2 & 6.5 & 129 & 31 & 24.0 & 0.03 \\
\hline
\end{tabular}

SRCC: signet-ring cell carcinoma; LNM: lymph node metastasis.

\section{Discussion}

The characteristics of early gastric SRCC, including LNM, clinicopathological features and prognosis, are still in dispute $[4,8,15]$. To achieve an agreement on the treatment options of early gastric SRCC, these aspects need to be further explored. Several studies had reported that risk factors for LNM in early gastric SRCC, tumor diameter is greater than 
$2 \mathrm{~cm}$, submucous infiltration and lymphatic vascular infiltration are risk factors and independent risk factors for LNM [15].

Table 7. Clinicopathological characteristics in 2 cases with lymph node metastasis for expanded indication.

\begin{tabular}{lll}
\hline Clinicopathologic Feature & Case1 & Case2 \\
\hline Age (year) & 41 & 56 \\
Gender & Female & Female \\
Location & Antrum & Antrum \\
Gross pattern & $2 \mathrm{~b}$ & $2 \mathrm{a}$ \\
Tumor size (cm) & $1 \mathrm{~cm}$ & $0.5 \mathrm{~cm}$ \\
Invasion depth & $\mathrm{m}$ & $\mathrm{m}$ \\
Lymphovascular invasion & 0 & 0 \\
H $\boldsymbol{p}$ infection & 0 & 0 \\
Atrophic gastritis & 0 & 0 \\
Ulcer finding & 0 & 0 \\
\hline
\end{tabular}

$H p$ : helicobacter pylori.

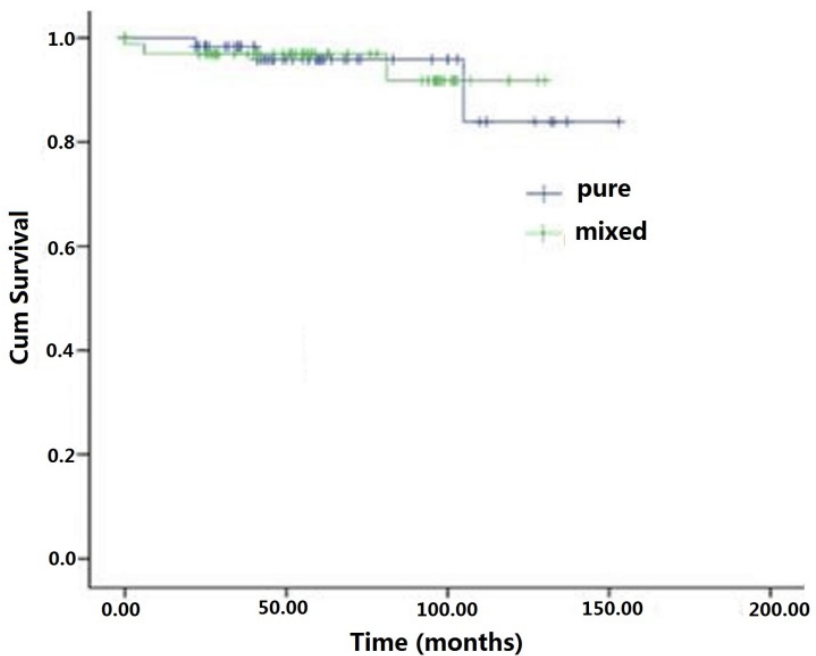

Figure 1. Overall survival curves of early signet ring cell gastric carcinomas patients underwent radical gastrectomies combined with lymph node dissections.

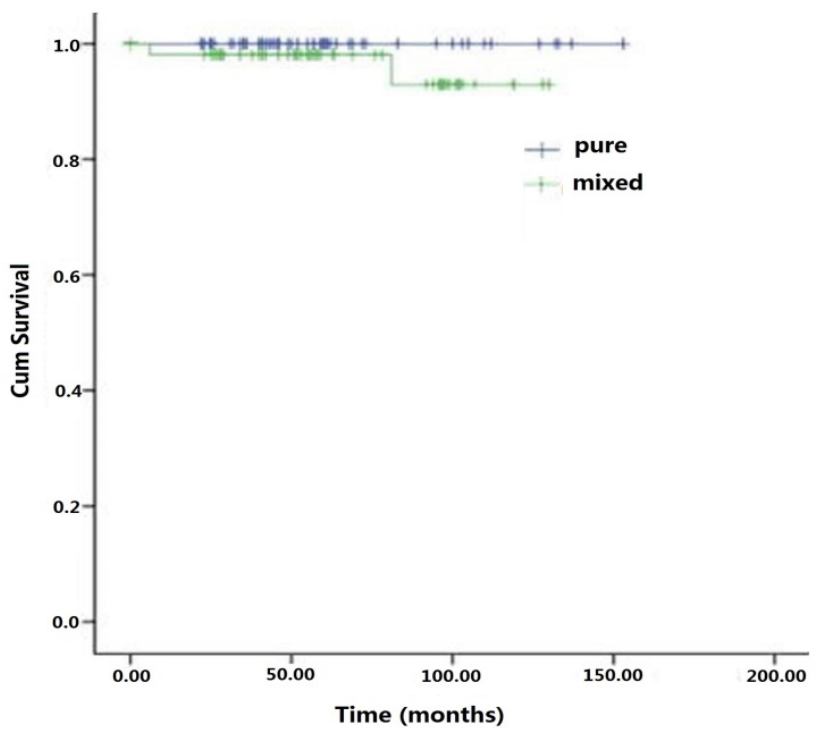

Figure 2. Specific survival curves of early signet ring cell gastric carcinomas patients underwent radical gastrectomies combined with lymph node dissections.

In our research, the risk factor for LNM in patients with pSRCC was LVI. In addition, we found that patients of pSRCC with high LNM rate were younger $(\leq 65,15.9 \% ;>65,0)$, had larger tumor size $(2-2.9 \mathrm{~cm}, 3 / 16,18.8 \% ;>3 \mathrm{~cm}, 3 / 22,13.6 \%)$, deeper invasion (SM, 5/28, 17.9\%), although there was no difference in statistics. With respect to the risk factors for LNM in mSRCC, our finding demonstrated that patients with larger tumor size $(>2 \mathrm{~cm})$ and more LVI are more likely to have LNM. At the same time, we observed that patients with mSRCC of LNM have earlier ages $(\leq 65,29.7 \%$; $>65,17.6 \%)$, more atrophic gastritis $(17 / 49,34.7 \%)$ and deeper invasion depth $(\mathrm{M}$, 8/42, 19.0\%; SM, 14/39, 35.9\%) despite unremarkble statistics differences. We think that the reason for this phenomenon is mSRCC have thin gastric mucosa because of atrophic gastritis, and larger tumor load because of larger tumor size, tumor cells easily penetrate the mucosa to the lymph and blood vessels of the lamina propria and grow LNM. On the other side, the deeper depth of invasion, the tumor cells are closer to the lymph and blood vessels of the lamina propria, and the LNM is more likely to occur. Patients with LNM are more frequent in younger patients whether pSRCC or mSRCC probably because of the rapid growth of the tumor cell. In our study, the mixed components of mSRCC included 19 moderate differentiated and 62 poorly differentiated histologic types. Although there is no significant difference in LNM, previous studies have suggested that the LNM of poorly differentiated gastric adenocarcinoma is higher and the prognosis is poorer [16], so surgical treatment is strongly recommended.

Our study showed that the lesion features such as male gender, a depressed gross type, $\mathrm{Hp}$ infection and LVI in the mSRCC group were more common than in the pSRCC group. The histology with mSRCC has been reported as one of the independent risk factors of LNM in EGC [17]. Our results demonstrated the previous studies that more LNM occured in mSRCC than in pSRCC $(27.2 \%$ vs $13.9 \%)$ and athough there was no significant difference between two groups. In consistent with the finding of previous reports [12], the factors that revealed more aggressive biologic characteristics in $\mathrm{mSRCC}$, such as positive LVI, and positive LNM, were more prominently associated with the mSRCC group than pSRCC group in EGC. Zheng et al. [11] explained the reason for the aggressive features in mixed-type gastric cancer was that $\mathrm{mSRCC}$ have more aggressive behavior such as proliferation, apoptosis, angiogenesis, mucin secretion, and cell adhesion according to increased expression of proteins such as Ki-67, EMMPRIN (extracellular matrix metalloproteinase inducer), and VEGF (vascular endothelial growth factor), which are involved in the angiogenetic process and cell proliferation in mixed-type gastric cancer. Park et al. 
[18] showed that mixed-type gastric cancer frequently showed CpG island hypermethylation. So, the mixed-type gastric cancer seems to be more aggressive than the pure-type. In addition, our study found that more male gender patients in mSRCC probably because men have bad habits of smoking and drinking, which are high risk factors for the occurrence of gastric cancer. We also discovered more depressed gross patterns, $\mathrm{Hp}$ infection in mSRCC may be related to ulcerative lesion and aggressive features. We thought that the mixed type of poorly differentiated histology is closely connected with strong aggressive behaviors and poor prognosis of mSRCC.

In agreement with the results of other studies, this research indicated that SRCC in EGC have a good prognosis [19]. Several studies have reported some factors contributing to the better prognosis of early gastric SRCC. Gronnier et al. [20] suggested that it is associated with younger in age. Kim et al. [19] reported that it is related to early discovery, the lesion is endoscopically concave, rich mucin protein in the cytoplasm and eccentric nucleus, the cancer cells are easily detected with pathological examination. In addition, the lower lymph node metastasis rate contributes to the better prognosis. Our research showed that early gastric SRCC either pure or mixed histology had no significant differences in the overall survival rate and the specific survival rate after the gastrectomy and lymph node dissection. However, the therapy options of mSRCC should be carefully considered based on characteristics of stronger invasiveness and higher LNM rate.

Recently, endoscopic therapeutic techniques, such as EMR or ESD, have been widely accepted as an alternate treatment to keep the quality of life for a subgroup of EGCs [21]. Technically, endoscopic therapy is used to resect the mucosa or the submucosa layer, without regional lymph nodes dissected. Thus, recognizing patients with high risk of LNM is significantly crucial for the application of endoscopic therapy. Our study showed that early gastric pSRCC had low LNM rate and good prognosis, early gastric mSRCC had relative high LNM rate and more aggressive features. Taking all these data into account, we can predict the therapy program of early gastric SRCC. Early SRCC, whether pure or mixed, has lymphatic vascular invasion, larger tumor size and deeper invasion depth, which are not suitable for endoscopic resection. However, it is considered that early mSRCC has more aggressive behaviors and should be much thought carefully for endoscopic resection.

In the current study, patients with out of indication had higher LNM rate than expanded indication whether pSRCC or mSRCC. As the sample size expands, we can find that there is a significant statistical difference between the expanded indication and out of indication $(P=0.03)$. We will speculate that the difference between them is more obvious when the number of samples is increasing. Therefore, patients who are not satisfied with the expanded indications of endoscopic resection have a higher LNM risk, gastrectomy combined with lymph node dissection is highly recommended. Of course, we should also carefully evaluate the indications of endoscopic resection, because our study found that 2 cases of pSRCC met the expanded indications had LNM and their size were not more $1 \mathrm{~cm}$, although the cases was fewer.

There are several main limitations in our study: 1) it was a retrospective study with a non-randomized design, selection bias was, inevitably, exist. But, we used a stringent study protocol with a consecutive patient selection procedure and a uniform exclusion method to minimize selection bias; 2 ) the variation in the number of lymph nodes retrieved was unavoidably present because of inconsistent surgical methods of lymphadenectomy among surgeons. However, the average number of lymph nodes studied in the cohort was high. We believe that any analysis on the risk of LNM in early gastric SRCC would have similar outcomes; 3) relatively short follow-up time. Therefore, a mass scale, multicenter, prospective and comparative cohort study is essential to verify the importance of the strategy.

Despite above problems, we think this study still has its own values: 1) a relatively large number of consecutive early gastric SRCC surgical resection cases $(\mathrm{N}=160)$ specifically for comparison of risk factors of LNM and clinicopathological features between mSRCC and pSRCC, which is rare; 2) in the analysis of risk factors for LNM and comparison of clinicopathological characteristic, the factors included in this study were more than previous studies, such as $\mathrm{Hp}$ infection, atrophic gastritis and ulcers; Comparison of LNM between expanded indication and out of indication was instructive to choices of treatment; 3) there are more factors leading to the formation of mSRCC and help us to explore the mechanism of its development; 4) unified implementation with the most rigorous investigation protocol by professional and skilled gastrointestinal pathologists guided with the WHO diagnostic code on early gastric SRCC. In conclusions, by analyzing risk factors of LNM, clinicopathological features and prognosis of pSRCC and mSRCC, it improves awareness of higher rate of LNM and more aggressive features in tumors with mixed histology of SRCC, 
helps to guide the endoscopic treatment of early gastric SRCC.

\section{Acknowledgements}

This work was supported by grants from the National Natural Science Foundation of China (Grant Nos. 81572338 and 81672380, 81201909, 81602089), the Nanjing Medical Science and Technology Development Program (Nos. YKK12072, YKK15061 and YKK16078). This work was also part of a C-class sponsored research project of the Jiangsu Provincial Six Talent Peaks (WSN-078).

\section{Ethical statement}

All procedures followed were in accordance with the ethical standards of the responsible committee on human experimentation (institutional and national) and with the Declaration of Helsinki and later versions. The exemption from the informed consent requirement is permitted by the Ethics Committee of the Affiliated Drum Tower Hospital Affiliated to Nanjing University.

\section{Competing Interests}

The authors have declared that no competing interest exists.

\section{References}

1. Ishikawa $\mathrm{S}$, Togashi $\mathrm{A}$, Inoue $\mathrm{M}$, Honda $\mathrm{S}$, Nozawa $\mathrm{F}$, Toyama $\mathrm{E}$, et al. Indications for EMR/ESD in cases of early gastric cancer: relationship between histological type, depth of wall invasion, and lymph node metastasis. Gastric Cancer. 2007:10:35-38.

2. Huang Q, Fang C, Shi J, Sun Q, Wu H, Gold JS, et al. Differences in Clinicopathology of Early Gastric Carcinoma between Proximal and Distal Location in 438 Chinese Patients. Sci Rep. 2015;5:13439.

3. Lo SS, Wu CW, Chen JH, Li AF, Hsieh MC, Shen KH, et al. Surgical results of early gastric cancer and proposing a treatment strategy. Ann Surg Oncol. 2007:14:340-347.

4. Chiu CT, Kuo CJ, Yeh TS, Hsu JT, Liu KH, Yeh CN, et al. Early signet ring cell gastric cancer. Dig Dis Sci. 2011;56:1749-1756.

5. Gotoda T, Yamamoto H, Soetikno RM. Endoscopic submucosal dissection of early gastric cancer. J Gastroenterol. 2006;41:929-942.

6. Ha TK, An JY, Youn HK, Noh JH, Sohn TS, Kim S. Indication for endoscopic mucosal resection in early signet ring cell gastric cancer. Ann Surg Oncol. 2008;15:508-513.

7. Abe N, Watanabe T, Sugiyama M, Yanagida O, Masaki T, Mori T, et al. Endoscopic treatment or surgery for undifferentiated early gastric cancer? Am J Surg. 2004;188:181-184

8. Kunisaki C, Shimada $\mathrm{H}$, Nomura M, Matsuda G, Otsuka $\mathrm{Y}$, Akiyama $\mathrm{H}$ Therapeutic strategy for signet ring cell carcinoma of the stomach. Br J Surg. 2004;91:1319-1324.

9. Fang C, Shi J, Sun Q, Gold JS, Xu GF, Liu WJ, et al. Risk factors of lymph node metastasis in early gastric carcinomas diagnosed by WHO criteria in 379 Chinese patients. J Dig Dis. 2016;17:526-537.

10. Flejou JF. [WHO Classification of digestive tumors: the fourth edition]. Ann Pathol. 2011:31:S27-31.

11. Zheng HC, Li XH, Hara T, Masuda S, Yang XH, Guan YF, et al. Mixed-type gastric carcinomas exhibit more aggressive features and indicate the histogenesis of carcinomas. Virchows Arch. 2008;452:525-534.

12. Endoscopic Classification Review Group. Update on the paris classification of superficial neoplastic lesions in the digestive tract. Endoscopy. 2005;37:570-578.

13. Dixon MF. Gastrointestinal epithelial neoplasia: Vienna revisited. Gut. 2002:51:130-131.

14. Stolte M, Meining A. The updated Sydney system: classification and grading of gastritis as the basis of diagnosis and treatment. Can J Gastroenterol. 2001;15:591-598.

15. Zhang M, Zhu G, Zhang H, Gao H, Xue Y. Clinicopathologic features of gastric carcinoma with signet ring cell histology. J Gastrointest Surg. 2010;14:601-606.
16. Park JM, Jang YJ, Kim JH, Park SS, Park SH, Kim SJ, et al. Gastric cancer histology: clinicopathologic characteristics and prognostic value. J Surg Oncol. 2008;98:520-525.

17. Huh CW, Jung DH, Kim JH, Lee YC, Kim H, Kim H, et al. Signet ring cell mixed histology may show more aggressive behavior than other histologies in early gastric cancer. J Surg Oncol. 2013;107:124-129.

18. Park SY, Kook MC, Kim YW, Cho NY, Kim TY, Kang GH. Mixed-type gastric cancer and its association with high-frequency $\mathrm{CpG}$ island hypermethylation. Virchows Arch. 2010;456:625-633.

19. Kim BS, Oh ST, Yook JH, Kim BS. Signet ring cell type and other histologic types: differing clinical course and prognosis in T1 gastric cancer. Surgery. 2014;155:1030-1035.

20. Gronnier C, Messager M, Robb WB, Thiebot T, Louis D, Luc G, et al. Is the negative prognostic impact of signet ring cell histology maintained in early gastric adenocarcinoma? Surgery. 2013;154:1093-1099.

21. Hyung WJ, Cheong JH, Kim J, Chen J, Choi SH, Noh SH. Application of minimally invasive treatment for early gastric cancer. J Surg Oncol. 2004;85:181-185; discussion 186. 